To appear in J.M. Bishop and A.O. Martin (eds.), Contemporary Sensorimotor Theory, Studies in Applied Philosophy, Epistemology and Rational Ethics

\title{
The Explanatory Status of the Sensorimotor Approach to Phenomenal Consciousness, and Its Appeal to Cognition
}

\author{
J. Kevin O'Regan \\ Laboratoire Psychologie de la Perception - Universite' Paris Descartes, Paris, France \\ jkevin.oregan@gmail.com
}

\begin{abstract}
This paper starts by providing a succinct overview of the sensorimotor approach to phenomenal consciousness, describing its two parts: the part that concerns the quality of sensations, and the part that concerns whether or not such qualities are (consciously) experienced. The paper goes on to discuss the explanatory status of the approach, claiming that the approach does not simply "explain away" qualia, but that on the contrary, it provides a way of thinking about qualia that explains why they are the way they are, stimulates scientific paradigms and produces testable predictions. A final part of the paper examines the relation of the theory to radical enactivism, claiming that some kind of "higher order" cognitive mechanism similar to that used in Higher Order Thought theories of consciousness is needed to account for what is usually meant by being conscious of something.
\end{abstract}

Keywords: Qualia, phenomenal consciousness, radical enactivism, Higher Order Thought, sensorimotor theory.

\section{Introduction}

In this paper I will address two contentious points about the sensorimotor ap- proach to consciousness. These concern the explanatory status of the approach, and the role and nature of cognition in the approach. But first a quick summary of the main ideas in the approach.

\section{Quick Summary of the Sensorimotor Approach}

The "hard" problem of phenomenal consciousness is the problem of qualia, that is, of understanding what it might be about, for example, red-sensitive circuits in the brain that causes them to create a "red" feel rather than, say, a "green" or "onion flavor" feel, or 
even any feel at all. Another way of illustrating the problem is to ask: "What would we have to build into a robot so that it really felt the touch of a finger, the redness of red, or the hurt of a pain?" The sensorimotor approach ([1], [2]) answers these questions by thinking about experience in a

new way. The approach is not just a philosophical theory but has scientific value in that it has been successful in driving new research paradigms.

The approach starts by making a distinction, and invoking separate mecha- nisms, to explain (1) the experienced quality of a feel, and (2) whether or not a person is conscious of this quality.

\subsection{The Experienced Quality}

Instead of thinking of the experienced quality of a sensory experience as being generated somewhere in the brain, the sensorimotor approach contends that the quality is constituted by the set of objective laws concerning the interaction with the world that the experience involves. The objective laws linking actions to re- sulting sensory changes are called "sensorimotor contingencies" or "sensorimotor dependencies".

For example, the quality of tactile experience is determined by the laws that determine the changes of sensory input that occur when you move your body with respect to a stimulating surface: the softness of a sponge, for example, is constituted by the fact that when you press on the sponge it cedes under your pressure. The quality of auditory experience is determined by laws like the fact that when you approach a sound source, the amplitude of the sensory input increases, etc. The similarities and differences between experienced qualities are constituted by the similarities and differences in such sensorimotor dependencies.

In addition to explaining similarities and differences between qualities, the view also explains "what it's like" (in the expression of Nagel [3]) to have a sen- sory experience: Certain objective physical facts about real sensory interactions are unique to the classic five sensory systems of vision, hearing, touching, tasting and smelling, and do not apply to mental activities like thoughts or imaginings, nor to autonomic processes in the nervous system. These objective facts are bodi- liness, insubordinateness and grabbiness.

Bodiliness is the objective fact that any movement of the body immediately changes input coming from sensory recep- tors. Insubordinateness is the objective fact that sensory input can be changed by the outside world without voluntary control by the person. Grabbiness is the objective fact that sudden changes in sensory input channels automatically capture attention. Bodiliness, insubordinateness and grabbiness provide sensory experiences with a quality of imposing themselves on us, of partly escaping our voluntary control. Taken together, they can be put into correspondence with the notion of "reality" or sensory "presence" which is typical of the phenome- nal quality of sensory experiences. Bodiliness, insubordinateness and grabbiness, when plotted on a "phenomenality plot" 
predict the extent to which people ex- perience sensations as possessing this quality of having "something it's like" ([1], [4]).

\subsection{Consciousness of the Quality}

While sensorimotor contingencies determine the quality of an experience, in- cluding its sensory presence, a second ingredient is needed for that quality to be

perceived consciously. Here the sensorimotor approach appeals to a particular, hierarchical, form of cognitive access similar to that used in the "higher-order" theories (HOT) of consciousness ([5], [6]). Returning to the example of the soft- ness of a sponge, a person who was absent-mindedly washing the dishes with a sponge while they were talking to a friend would not at that moment be consciously experiencing the softness. Only if they cast their attention on and cognitively accessed the quality of their interaction with the sponge would they normally be said to be consciously experiencing the softness. I shall come back to this cognitive access later.

\section{Solving or Dissolving the Hard Problem?}

The trick used in the sensorimotor approach is to try to "dissolve" the hard prob- lem of qualia rather than "solving" it. The idea is that the so-called hard problem is only hard because the terms generally used to talk about consciousness are unclear. When consciousness is given a clear definition, the problem dissolves. In particular, as regards phenomenal experience, the sensorimotor approach sug- gests that if we think clearly about what we mean by having an experience, then we see that it involves, not something being generated in the brain, but a way of interacting with the world. The hard problem of finding a brain mechanism that generates experience is thereby eluded.

Note however that it is not the case that the sensorimotor approach "explains away" qualia, in the sense that it brushes them aside as a non-problem. It takes seriously the question of accounting for the experienced quality of sensation, and provides answers that are scientifically testable.

The case of softness is a case where this approach works well. Consider for example the "hard" question of how the softness of the sponge might be gener- ated by brain mechanisms. From the point of view of the sensorimotor theory, this question does not make sense, because the softness lies in the fact that if I press the sponge, it squishes under my pressure. It is a sensorimotor law. As an abstraction, it cannot be found anywhere in particular, and certainly it is not "generated" in the brain. (Of course the brain has a role to play, namely to enable all the mechanisms that allow the organism to be engaged with the sensorimotor laws of softness - but there will be many such mechanisms, and they will probably be widely distributed in the brain, since all sorts of motor control and sensory systems underlie our ability to interact in the "soft"-specific way. And no one, or combination of, these mechanisms could be considered to "generate" 
the softness feel.)

If we ask the "hard" question of why softness feels the way it does, the sen- sorimotor approach says that the answer boils down to the objective physical facts that describe the interaction we have with the sponge. The possibility of inverted qualities - the idea that somehow a soft sponge would feel hard and a hard sponge would feel soft - becomes inconceivable because what we mean by feeling softness by definition requires the sponge to react appropriately to our pressing, and this can only occur if objectively the sponge is soft.

And finally we could ask why softness has sensory presence at all, rather than providing an experience like, say, a thought or like imagining. For this the sen- sorimotor approach appeals to the concepts of bodiliness, insubordinateness and grabbiness. These supply potentially available facts about the current ongoing interaction which are constitutive of its "realness" or "presence". Because they have the consequence that our minds are subservient to the external stimulation, the quality of softness imposes itself upon us and manifests its presence in a way that other mental processes like thoughts, imaginings, memory or autonomic processes do not.

Thus, in the case of the softness of the sponge, important aspects of the explanatory gap dissolve, and we have an intuitively plausible way of accounting for not only the quality itself, but also its sensory presence.

However a priori it does not seem obvious that all sensations can be accom- modated under this view. In particular, sensations like those of colour and smell, and perhaps even auditory and passive tactile sensations do not seem to involve actively interacting with the world. It is nevertheless the wager of the sensori- motor theory to try to cover all sensations in this way. We do this because of the philosophical advantage the approach promises in bridging the explanatory gap.

And furthermore, when the idea is taken seriously, it leads to productive sci- ence: stimulating the discovery of change blindness, accounting for phenomena in color science, and promoting research in sensory substitution. In a way it is sur- prising that finding proper definitions should propulse new scientific advances. The reason is presumably that clear definitions allow the relations between ob- jective phenomena to be more clearly seen, revealing causes and effects that are otherwise obscured.

\section{The Explanatory Status of the Approach, and Anthropocentric Fears}

What then is the explanatory status of the sensorimotor approach? One should probably not say that the sensorimotor approach is a theory in the sense that it provides explanations. Rather, it is a way of thinking about the phenomena of consciousness that allows them to be divided into scientifically amenable bits, and which preserves a plausible link with some of the important ways we use the term in everyday life. 
Questions that seemed to demand explanations evaporate when we think in the new way.

By way of analogy, one could say that the sensorimotor account answers the question of phenomenal consciousness in a similar way to how the question of life was answered in modern biology. Instead of finding a magical vital spirit that generated life, modern biology discovered that it was a "category mistake" (cf. Ryle [7]) to look for a substance to generate life. We now conceive of life as being an abstraction used to describe organisms that interact in certain ways with their environments. By taking this stance, modern biology now decomposes the problem of life into the smaller problems of accounting for each of the as- pects of the way organisms interact with their environments. And each of these

subproblems becomes tractable using the normal laws of physics and chemistry. No magic vital essence is required. Of course poets will say that the magic of life is not fully captured.

Similarly the sensorimotor approach considers that it is a category mistake to search for a neural correlate that generates consciousness in the brain. Instead, the sensorimotor approach suggests that it is advantageous to more carefully define the different aspects of consciousness, and decompose it in two parts: the sensorimotor interactions whose laws constitute experienced quality; and a form of cognitive access which makes that quality conscious. Both parts are amenable to a biological explanation, and both parts keep a link with normal everyday notions of consciousness, with some limitations (see the section on ethical consequences).

The trouble is that just as it was difficult for many people to accept that modern biology could adequately account for the "mystery of life", many people resist the sensorimotor approach, and claim that it cannot dissolve the "mystery of qualia".

I suggest that in both cases however the problem lies not in the theories, but in an anthropocentric fear. At the beginning of the 20th Century people felt threatened by the idea that "life" might just be a chemical process. Today, people are threatened by the idea that "feel" is simply a way of interacting with the world. After all, humans are not the only agents that can interact with the world, and even machines could be said to do so. There is the terrible danger that feel, this very special property of sentient beings, might be usurped by ma- chines. Subconsciously it is therefore an excellent tactic to entertain a degree of artistic confusion about what consciousness is, and to resist pidgeonholing it in any rational way, and in particular in the way suggested by the sensorimotor approach. That way we reduce the chances that we will ever be labelled as ma- chines. Thus many scientists, and among them even roboticists and workers in artificial intelligence, believe that there is something fundamentally missing in our understanding of human feel, and which is preventing us building it into our machines today. I claim that this belief constitutes an unconsciously motivated diversion tactic to preserve our last bastion of humanity, namely feel, from ma- chines. It is a form of anthropocentric chauvinism, a 
hidden fear that secretly fuels an unconsciously maintained confusion.

\section{The Need for Cognitive Access}

If we accept the wager that sensorimotor laws will completely describe all as- pects of the phenomenal quality of our experiences, then another issue arises. Does the theory really need the second mechanism, namely the mechanism of cognitive access? Hutto \& Myin ([8]) consider that invoking cognitive access is a capitulation to intellectualist, cognitivist and representationalist theories. They recommend dispensing with cognitive access and propose taking a radical enac- tivist position in an account of consciousness that retains only the sensorimotor contingencies. More representation-friendly theorists, such as Block (cf. [9]) have

further intimated that in any case the appeal to cognitive access does not solve the explanatory gap problem, and sneaks in the concept of consciousness by the back door.

\subsection{Radical Enactivism}

There would appear to be two reasons for which a radical enactivist might want to deny the role of cognitive access in determining phenomenal consciousness. The first might be to claim that all cognition is ultimately based in sensorimo- tor interaction with the world. Thus the higher order form of cognitive access that the sensorimotor theory introduces to characterise consciousness of sensory qualities should be considered to be "enactive", non-representational processes. As we shall see in the next section, I have no objection to such a view, pro- vided it retains the notion that there is the enactive equivalent of "higher order" access that determines whether the quality of the interaction is experienced as conscious. Further, perhaps the higher and lower order access should not be considered as a dichotomy, but as two extremes in a continuum of access.

A second reason to deny the role of cognitive access in determining phenome- nal consciousness might be essentially a matter of definition. It might be argued that there are forms of phenomenal consciousness which do not require the per- ceiver to be cognitively occupied with ongoing sensorimotor activity. Rather, this sensorimotor activity could be considered to provide phenomenal conscious- ness in itself. Proponents of such a view might be motivated by the intuition that even when I'm talking to a friend while I wash the dishes, I am still in some sense experiencing the softness of the sponge. Further, presumably beings like newborn babies or animals with limited cognitive capacities let's say frogs - do really experience their worlds phenomenally. Even without welldeveloped minds, such beings nevertheless possess some kind of minimal, "organismic" form of feel ([10], [11]).

Taking this option of defining certain kinds of sensorimotor interactions to possess phenomenal consciousness in themselves, leads down a slippery slope. Consider a system like our digestive system, the immune system, or plants. One could also look at artificial 
systems like missile guidance systems or even the lowly thermostat. All these systems could be considered sensorimotor systems. They engage in sensorimotor interactions. One could say that they exercise sen- sorimotor laws. But surely they do not feel anything.

One could argue that such systems would begin to have experiences if one made them sufficiently complex. Perhaps the nematode worm c. elegans, which is probably a kind of biological automaton with a fixed number of cells and well- defined neural pathways has no feel, but a fly, or if not, perhaps a frog, or a mouse surely has some kind of feel. Intuitively this might be because it is not completely pre-wired, because it has a certain degree of neural plasticity and can learn from its past experiences, or because it is sufficiently complex. But why? What might it be about not being pre-wired, about plasticity or complexity that instills feel into a system? Or you could argue that it is simply what you mean by saying that a system has a feel, that the system displays a certain amount of adaptability,

flexibility and complexity. We could take this stance as a matter of definition for feel, but then all sorts of systems, be they biological, mechanical, economical, evolutionary, display flexibility, adaptability and complexity, and yet presumably they don't feel. Perhaps certain biological systems (namely ourselves) have an extra special degree of flexibility, adaptability and complexity. But it is not clear what exactly this extra special degree is, nor how to characterise it in a way that makes it evidently constitutive of feel.

Finally another tactic might be to conjecture that in order to provide such special adaptability, flexibility and complexity, some kind of special brain sys- tems are necessary, and these produce feel as a by-product. An obvious candidate would be systems involved in drives, emotions or arousal. But such arguments are precisely the arguments that the sensorimotor theory was trying to avoid. They hark back to the "category mistake" of thinking that feel is a substance produced by the brain, and they immediately face the explanatory gap problem: how could one logically ever explain how such emotional or arousal systems might create feel. Whatever chemical or neural mechanism was postulated, be it some neuromodulator in the brain that labels states as being emotionally charged, or be it the likes of oscillations, neural synchronisation, reentrant loops, or even quantum gravity mechanisms in microtubules, one would have to explain pre- cisely what it is about this mechanism that made it "like something" to feel.

The solution proposed by the sensorimotor approach is therefore to reject the existence of an "organismic" kind of feel that requires only non-cognitive sensorimotor interactions, and to claim that sensorimotor interactions are only part of what we mean by experiencing. Certainly their qualities constitute the experienced phenomenal qualities. But a second mechanism, namely a form of higher order cognitive access, is additionally required to make the experienced qualities conscious. In fact as we shall see below, it could be argued that what I call higher order cognitive access is actually just a way of being a little more precise about the notions of adaptability, flexibility and complexity which intu- itively we want to have in our definition of consciousness. In any case I 
maintain that something resembling cognition, as well as the idea of different modes of access to ongoing interactions, are necessary to capture a satisfactory definition of what we generally mean by consciousness.

\subsection{Adopting a Form of Higher Order Thought}

To support the idea that some notion of higher order cognition is essentially what people normally mean when they say they are conscious of something, let us consider again the situation where I am washing the dishes with a sponge. Let us try to capture what it means to say that I'm conscious of the softness of the sponge.

What first comes to mind is to say that:

(A): In my current rational behaviour (such as deciding or planning actions, possibly making linguistic utterances), I am making use of the fact that I can currently confirm that the sponge is soft.

We shall see that this is not sufficient and needs further development, but let us use this as a working start. And let us decompose each of the underlying statements, starting at the end.

Saying that I can currently confirm that the sponge is soft means that I can confirm that when I press it, it squishes. But there are many ways of press- ing, and many ways of confirming that it squishes, involving different muscle combinations, finger positions, etc., each involving different neural signals. The details are not relevant however, because my brain has abstracted a category called "soft", and the current sensorimotor activity fits this category. Further- more when I say: "I can confirm...", this does not mean that I as a person am aware of how this abstraction has been effected, nor does it mean that I am aware of the intimate neural, muscular or sensory workings involved. On the contrary, it is the case that my brain must have made this abstraction, and must now be confirming that its conditions are fulfilled. In past papers, I have described this situation by saying that the brain must "currently be exercising its mastery of the sensorimotor contingency of softness".

So to be more precise, we should modify (A) as follows:

(A'): In my current rational behaviour (such as deciding or planning actions, possibly making linguistic utterances), I am making use of the fact that my brain is currently exercising mastery of the sensorimotor contingency of softness.

Note also that because softness is an abstract sensorimotor law, statement (A') says that I am making use of "the fact" that I can confirm that the sponge is soft: I'm not making use of a set of neural commands, or an ongoing pattern of neural activities or muscular giveand-takes: I am making use of the fact that a condition has been satisfied. 
Now let us analyse more closely what it means to be "making use, in my rational behaviour" of this fact. It means that the fact-of-softness is playing a role in this behaviour. For example, I have grasped the sponge in order to wipe the plate, rather than trying to use the plate to wipe the sponge. Dishwashing is a rational behaviour, requiring, among other things, to be able to use the softness to appropriately direct sponge-use.

But notice that I could be doing the dishwashing as I'm absorbed in following an interesting TV programme. Although it would be true to say that I'm making rational use of the sponge's softness, under normal parlance one would not say I was conscious of the softness of the sponge. Furthermore one could imagine a dishwashing robot that appropriately chose sponges to wash dishes (rather than the other way around), doing so by detecting that the sponges are soft whereas dishes are hard. Statement (A') would then apply to the robot, but in normal parlance we would not say that the robot was conscious of the softness.

For us to be more willing to admit that the robot was conscious of the softness, it would have to be not only making use of the softness in its dishwashing, but, as suggested in HOT theories ([5], [6]), it would have to know it was doing this. This kind of knowing would have to be of a fairly high level: thus it would not be sufficient if the robot just wrote out on its terminal "Currently checking softness to choose between sponge and plate...", since this kind of statement could be

generated automatically by its dishwashing routine. To appear conscious, the robot would have to give us evidence that it knew what it was doing in a wider context. It should "potentially have in mind" that there are a variety of other things that it might have been mentally accessing instead of the softness: and these things would not only be those that are part of the dish-washing routine like the hotness of the water, the size of the plates, etc.; they would include contextual things like the sound of the television in the background, the tiredness in its limbs; and even more general thoughts or attitudes the robot might have towards what it is doing and its current situation, like the fact that today there are more dishes than usual, or that it might be more pleasant to be reading a book, etc. I suggest that in normal parlance, being conscious of the softness of the sponge involves not just attending to the softness of the sponge, but doing so in a way that includes knowing that potentially one could also attend to a variety of related things that are "latent" or in the "fringe" of one's awareness.

Let us call this "wider" type of "making use in one's rational behaviour of X": "having higher order cognitive access to X". This notion of higher order cognitive access thus implicitly includes not just $X$, which is the central thing actually being "made use" of, but also a range of other "latent" things in the "fringe", say W,Y,Z..., and that pertain to one's current situation, to the current context, or to one's thoughts or attitudes towards $\mathrm{X}$, and which can potentially also be "made use" of.

Then, a better approximation than (A') to what I mean by being conscious of the softness 
of the sponge is:

(B) I have higher order cognitive access to the fact that my brain is currently exercising mastery of the sensorimotor contingency of softness.

In [1] I had formulated (B) by employing the term "cognitive access" in a hierarchical way: I had said that what I mean by being conscious of the softness of the sponge is "Cognitively accessing the fact that I am cognitively accessing the fact that I am currently exercising mastery of the sensorimotor contingencies of softness." Though defensible with the appropriate definition of "cognitive access", this usage has led to misunderstandings, because the higher and lower levels of cognitive access are different in the degree to which they are properly cognitive, and in the type of "latent" or "potential" facts that they concern. It would be interesting elsewhere to further clarify these issues, but here I wish to consider what seems like a much more important question.

\subsection{Obtaining Phenomenality}

Even if we accept (B) as a good approximation to what it means to be conscious of the softness of the sponge, there seems still to be a problem. Being conscious of the fact of softness is not the same as consciously and phenomenally experiencing the softness. Being conscious of a fact is phenomenally quite a different thing from consciously feeling. Feeling involves a phenomenal aspect, whereas the fact does not. How could having higher order cognitive access to a sensorimotor law provide an actual feel?

Indeed this is an issue that is skirted by HOT theories - these simply assert that "a mental state is conscious just in case it is accompanied by a higher-order thought to the effect that one is in that state" ([12] p. 352). From the sensori- motor point of view such a statement first is confusing, because mental states are not conscious, people are conscious. Second, while having such HOTs seems intuitively to be what we mean when we say we are conscious of a fact, some- thing is missing when we are talking about a phenomenal experience, namely the experienced phenomenal quality of the presence of the environment.

The missing thing is provided by the sensorimotor approach. There are two ideas. The first depends on the fact that when you are cognitively accessing an ongoing sensorimotor interaction, you are not just accessing a single thing about that interaction. Among the battery of facts $\mathrm{W}, \mathrm{Y}, \mathrm{Z}$... mentioned above and which are all potentially accessible, some contribute to providing an impression of "being connected to" or "in intimate contact" with the sensorimotor interaction that is going on. This is part of what provides the special impression of phe- nomenal modality and presence. The second idea concerns the special role that "grabbiness" plays in dominating our mental functioning, and thereby generating a form of subservience to our environments, which also strongly contributes to the impression of sensory presence or reality, of sensory experiences. 
Phenomenal Modality and Presence. The main fact that you are cog- nitively accessing when you are experiencing the softness is that the current interaction obeys the laws of softness. However you are not simply cognitively accessing the fact of softness, which you could also get in a different fashion, say, by reading a book. There are additional facts available, such as for example that you are obtaining the feel of softness through a tactile interaction, and not through visual or auditory interactions - you have access to sensory modality ([13]). You can know this about your mode of access because you can access the fact that your finger motion is having an effect on sensory input. Another potentially available fact is that the interaction involves, say, your right hand, and these particular fingers. Such facts, of which there are very many, are not currently in the main focus of your attention. They are examples of the "latent" facts in the fringe of your awareness that you could, if you wanted, immediately make use of by bringing them into attentional focus. These potentially available facts are nevertheless part and parcel of the main fact, namely the fact of soft- ness, and they contribute to the impression that the softness is of a type obtained through touch.

An even more important set of facts that you have potential access to are those that determine the impression of sensory presence of the softness. These facts are the bodiliness, insubordinateness and grabbiness of the ongoing interaction.

Bodiliness consists in the fact that moving your body (in particular your fingers) is what is currently providing changes in sensory input. This is a typical fact about sensory experiences deriving from the outside world, and it is not true of mental states like thoughts, memory or hallucinations, nor is it true of internal visceral states.

Insubordinateness is also an indicator that you are really currently interacting with the world: it consists in the fact that although

at this moment most of your changes in sensory input are coming from the changes caused by moving your fingers, there is an element of unpredictability: there is always the possibility that something might brush your fingers without you moving them (an insect inside the sponge?). Finally, and most important is that you have potential cognitive access to the grabbiness of the interaction. Grabbiness is the fact that it is possible that while you are engaged in exploring the sponge, your attention might be grabbed incontrovertibly by some sudden occurrence which is beyond your control: for example if you encounter a pin in the sponge that pricks your finger. Grabbiness is a fundamental property of sensory systems. Unlike thoughts and visceral states, sensory systems are hard- wired so that they can interrupt cognitive processing and orient attention to sudden sensory changes (see [1], [4]).

Bodiliness, insubordinateness and grabbiness are facts about your current sensorimotor interaction which are also part of the fringe of your higher order cognitive access to the softness. They are proofs that the current interaction is of an ongoing kind, that it is occurring with the outside world and is not purely a mental creation or some kind of visceral functioning. The potential to access these facts procures the feeling of modality 
(tactile, not visual or other), the "realness", and the sensory "presence" of the interaction.

Presence and Subservience to the World. It is important to note the special role played by grabbiness. Contrary to what happens in normal thinking where our thoughts are more or less under our own control, in the case when we are involved in sensorimotor interactions with the world, because of grabbiness, our cognitive processes are partially subservient to the interactions. Because sensory systems are hard-wired to be able to interrupt our cognitive processing, we partially lose our mental control, and our attentional and cognitive capacities are drawn along and directed incontrovertibly by the ongoing interaction. Thus when we have higher order cognitive access to a sensorimotor interaction, not only are we aware of the battery of facts that correspond to the quality of that interaction, but our cognitive processes themselves are partially controlled and dominated by what is happening in that interaction. This potential direct effect of sensory stimulation on ongoing mental processing is an another important factor that gives sensory stimulation its specific feel of presence or reality.

In summary, when we have a sensory experience, the feeling we have of on- going stimulation, of something occurring to us, of having an outside source of stimulation, can be subdivided into many sub-aspects such as those above. When I say it feels like something rather than nothing to have a sensory ex- perience, this statement can be decomposed into being conscious of facts like: it's modal (because it has the properties of touch, not of other senses), it has a particular quality (determined by the particular sensorimotor law), it's real (because it has the bodiliness, insubordinateness and grabbiness of real interac- tions, and furthermore controls my thoughts the way real things do), etc. The claim is that there is nothing more to phenomenal consciousness than potential conscious access to all these facts.

Note defining conscious phenomenal experience as having higher order access to a sensorimotor interaction provides an improvement on HOT theories by giving an intuitively satisfactory account of the phenomenal aspect associated with conscious sensations. Of course in line with radical enactive approaches one could say that the "cognitive access" that I invoke does not have to be played out in a representationalist way. But some form of higher order "access" is necessary, simply because that is the normal usage of the word "conscious".

\section{Conclusion}

The sensorimotor approach is not a theory, but a way of being precise first about what we generally mean by the quality of an experience, and second about what we generally mean by being conscious of an experience.

As regards sensations, the approach proposes that experiencing a sensation involves being engaged in a sensorimotor interaction. The sensorimotor contin- gencies that describe that interaction constitute the experienced qualities of the experience. Far from "explaining 
away" the problem of qualia, the sensorimotor way of thinking provides immediate links between how we describe our sen- sory experiences (they have ineffable similarities and differences between their qualities, they are modal, they have sensory presence or realness...) and physical properties of the sensorimotor interactions involved (the sensorimotor laws, their bodiliness, insubordinateness and grabbiness...).

As regards what we mean by being conscious of something, the approach proposes that normal parlance requires appeal to a form of "higher order" cog- nitive access. This does not involve magical mechanisms and does not "sneak in" consciousness by the back door.

Unlike HOT approaches, the question of how higher order access to sensori- motor interactions can be accompanied by phenomenality receives an intuitive answer in terms of potential access to a battery of latent facts about the ongo- ing sensorimotor interactions, and in terms of the grabbiness of sensory systems, which creates a special subservience of mental states on the outside world.

Interestingly taking these definitions leads to some important theoretical, ter- minological and ethical questions. The ethical questions derive from the fact that the term "being conscious of something" demands the mental faculties needed for "higher order" access. If we want the term to retain the same meaning for different creatures, then we have to admit that consciousness, in this definition, must be present in progressively lesser degrees in children, in new-born babies and animals, whose mental faculties are less. This leads to the uncomfortable conclusion that creatures with lesser mental capacities do not consciously expe- rience, for example, pain, in the same way human adults do, if at all. Trying to appeal to some other, "organismic" form of consciousness that all living creatures possess is not a defensible option, I have argued. The conclusion is that consciousness is a matter of degree, and so not an effective criterion to determine animal rights and whether we should give anesthetics to babies. We have to look elsewhere for reasons to justify our ethical practices.

Acknowledgements. I warmly thank Erik Myin, and particularly Jan Degenaar for help in clarifying this manuscript.

\section{References}

1. O'Regan, J.K.: Why red doesn't sound like a bell: Understanding the feel of consciousness. Oxford University Press, New York (2011)

2. O’Regan, J.K., No“e, A.: A sensorimotor account of vision and visual consciousness. Behav. Brain Sci. 24(5), 883-917 (2001)

3. Nagel, T.: What is it like to be a bat? Philos. Rev. 83, 435-456 (1974)

4. O'Regan, J.K., Myin, E., No"e, A.: Towards an analytic phenomenology: the con- cepts of 'bodiliness' and 'grabbiness'. In: Carsetti, A. (ed.) Proceedings of the International Colloquium: Seeing and Thinking. Reflections on Kanizsa's Studies in Visual Cognition, University Tor Vergata, Rome, June 8-9, pp. 103-114. Kluwer (2004) 
5. Rosenthal, D.M.: From Rocco Gennaro (ed). In: Higher-Order Theories of Consciousness: An Anthology, pp. 17-44. Johns Benjamins Publishing Company, Amsterdam (2004)

6. Carruthers, P.: Higher-Order Theories of Consciousness. In: Zalta, E.N. (ed.) The Stanford Encyclopedia of Philosophy (Fall 2011 Edition) (2011)

7. Ryle, G.: The concept of mind. University of Chicago Press (1949)

8. Hutto, D.D., Myin, E.: Radicalizing enactivism: Basic minds without content. MIT Press, Cambridge (2012)

9. O'Regan, J.K., Block, N.: Discussion of J. Kevin O'Regan's 'Why Red Doesn't Sound Like a Bell: Understanding the Feel of Consciousness'. Rev. Philos. Psychol., 1-20 (2012)

10. Gallagher, S.: Philosophical conceptions of the self: implications for cognitive sci- ence. Trends Cogn. Sci. 4(1), 14-21 (2000)

11. Thompson, E.: Mind in life: Biology, phenomenology, and the sciences of mind, vol. xiv. Belknap Press/Harvard University Press, Cambridge (2007)

12. Rosenthal, D.M.: Consciousness and mind. Oxford University Press (2005)

13. Clark, A.: A Case where Access Implies Qualia. Analysis 60(265), 30-37 (2000) 